\title{
TRANSIENT BEHAVIOUR AND STABILITY FOR THE THERMOELASTIC CONTACT OF TWO RODS OF DISSIMILAR MATERIALS
}

\author{
J. R. Barber and RongGang Zhang \\ Department of Mechanical Engineering and Applied Mechanics, University of Michigan, Ann Arbor, \\ MI 48109, U.S.A.
}

(Received 14 March 1988)

\begin{abstract}
The paper investigates the transient behaviour and stability of a system consisting of two thermally conducting elastic rods in contact on their end faces, the other ends of the rods being built-in to two rigid walls which are maintained at different temperatures. It is assumed that there is a thermal resistance at the interface between the rods which is a known function of contact pressure or gap.

A perturbation method is used to analyse the stability of the system and it is shown that there is a range of conditions under which the steady-state solution is unique but unstable. A finite difference method is then used to model the transient behaviour; it shows that under such conditions the system always tends to a steady oscillatory state in which the contact pressure varies periodically with time, possibly with periods of separation.
\end{abstract}

\section{INTRODUCTION}

It is well known that mathematical difficulties can arise in the solution of steady-state thermoelastic contact problems if conventional idealized boundary conditions are applied [1-3]. Difficulties over existence of solution can be circumvented by postulating a more physically realistic boundary condition involving a pressure or gap dependent thermal resistance at the interface $[4,5]$, but multiple solutions are still possible with this formulation.

The stability of the steady-state solutions has been investigated for various onedimensional systems which exhibit multiple solutions, using perturbation methods [6-8]. In all cases, it was found that when the steady-state solution was unique, it was also stable, whereas when multiple solutions were obtained, they were alternately stable and unstable. Furthermore, in each case a variational statement of the stability criterion could be formulated which was mathematically equivalent to that prescribed by the perturbation solution, but it proved impossible to justify the variational formulation from first principles in view of the non-conservative nature of the system.

More recently, an investigation into the stability of the two dimensional contact of two half-planes [9] has shown that the behaviour is considerably more complex than previous results suggested. The problem was solved by examining the conditions under which a sinusoidal perturbation in the contact pressure can grow with time, following techniques developed by Dow and Burton [10] and Richmond and Huang [11]. As in the onedimensional cases cited above, a characteristic equation is obtained whose zeros correspond to the exponential growth rates of physically admissible perturbations. However, in contrast to the previous solutions, the characteristic equation involves the thermal diffusivities of the two materials as well as the 'steady-state' properties such as conductivity and thermal expansion coefficient. It is therefore possible to envisage material combinations for which the steady-state solution-determined by steady-state properties-is unique, but unstable.

The transient behaviour in such cases remains to be determined, but the most likely possibility would seem to be some kind of non-linear oscillation about the unstable steady state. This conclusion has important consequences for the interpretation of previous solutions of steady-state thermoelastic contact problems, such as the classical thermoelastic Hertz problem [12], which have always been implicitly assumed to be stable.

If one of the half-planes in [9] is replaced by a non-conducting rigid body, the characteristic equation reduces to a simpler form and the stability criterion shares many of the features of those from earlier one-dimensional analyses. We therefore conclude that the 
possibility of a unique but unstable steady state requires that the two contacting bodies should both be thermal conductors. In fact, the instability can be directly traced to a difference in the thermal diffusivities of the materials, which govern the rate at which they separately respond to perturbations in boundary conditions.

In the present paper we shall demonstrate that the same features can be obtained in a onedimensional system consisting of two contacting rods, for which the transient problem is sufficiently tractable numerically to permit the resulting oscillatory behaviour to be investigated. In fact, this system is closer to that originally proposed by Comninou and Dundurs [13] as an appropriate model for the investigation of stability problems in thermoelastic contact. One of the present authors [J.R.B.] must take responsibility for making the further idealization to a single rod model [6], thereby delaying the discovery of the present more complex behaviour by seven years!

\section{THE MODEL: STEADY-STATE SOLUTION}

The system is illustrated in Fig. 1. Two perfectly conducting rigid walls, separated by some distance, are maintained at temperatures $T_{1}$ and $T_{2}$. Two uniform elastic rods of lengths $L_{1}$ and $L_{2}$ are built into the respective walls, leaving a gap $g_{0}$ between the free ends when the temperature everywhere is zero. The thermal conductivity, diffusivity and coefficient of thermal expansion of the rod materials will be denoted by $K_{i}, k_{i}$ and $\alpha_{i}(i=1,2)$ respectively.

Heat flow can occur between the free ends of the two rods across a thermal resistance $R$, whose value is assumed to be some function of the contact pressure or the gap. No assumption is made at this stage about the nature of this function, although on physical grounds it is expected to fall monotonically as the gap is reduced or the contact pressure increased. The resistance function is discussed in more detail in section 5.2 below.

The steady-state analysis is very similar to that for the one-rod model, enabling some of the elementary steps to be omitted here. The reader is referred to [6] for details. In the steady state, the temperature varies linearly along each rod and the system essentially consists of three thermal resistances in series. Thus, if the contact resistance, $R$, is known, the temperature $T_{i}^{0}$ of the free ends of the rods can be determined from continuity of heat flux, giving

$$
T_{i}^{0}=\frac{R T_{i}+R_{1} T_{2}+R_{2} T_{1}}{R+S}
$$

where $R_{i}=L_{i} / K_{i}$ is the thermal resistance of $\operatorname{rod} i$ and $S=R_{1}+R_{2}$.

The free thermal expansion is then

$$
u_{i}^{\text {th }}=\frac{1}{2} \alpha_{i} L_{i}\left(T_{i}+T_{i}^{0}\right)
$$

and the gap is reduced to

$$
g=g_{0}-u_{1}^{\mathrm{th}}-u_{2}^{\mathrm{th}} .
$$

Substituting for $u_{i}^{\text {th }}$ and $T_{i}^{0}$ from equations (1) and (2), we obtain

$$
f(g) \equiv\left(1+\frac{R}{S}\right)^{-1}=\frac{g-g_{1}}{\frac{1}{2} \alpha L\left(T_{1}-T_{2}\right)}-\frac{2 \alpha_{2} L_{2}}{\alpha L},
$$

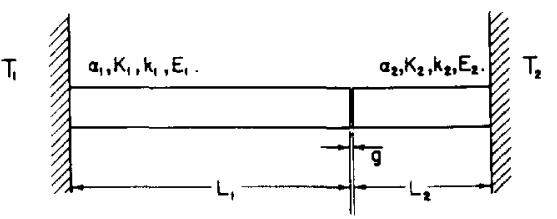

Fig. 1. Geometry of the two rod system. 
where

$$
g_{1}=g_{0}-\left(\alpha_{1} L_{1}+\alpha_{2} L_{2}\right) T_{1}
$$

is the gap which would occur if $T_{2}=T_{1}$ and

$$
\alpha L=\left(\alpha_{1} L_{1} R_{1}-\alpha_{2} L_{2} R_{2}\right) / S .
$$

As in previous papers [6-8], the above analysis can be generalized to the case of contact by defining

$$
g=-\left(\frac{L_{1}}{E_{1}}+\frac{L_{2}}{E_{2}}\right) p ; \quad p>0, g<0,
$$

where $p$ is the contact pressure.

In equation (4), we note that the contact resistance $R$ is a positive, monotonically increasing function of $g$, in both contact and separation regions. The function $f$ is therefore also a monotonic but decreasing function in the range of $0<f<1$.

An equation of similar form to (4) was found to govern the steady-state solutions of previous one-dimensional thermoelastic contact systems [6-8]. In particular, we recall that multiple solutions will be predicted when the straight line defined by the right hand side of equation (4) has a sufficiently large negative slope, corresponding to a critical value of $\alpha L\left(T_{2}-T_{1}\right)$. The direction of heat flow needed to permit multiple solutions depends on the sign of $\alpha L$ and hence on the relative lengths of the rods [see equation (6)]. In particular, we note that the solution will always be unique if

$$
\alpha L\left(T_{1}-T_{2}\right)>0 .
$$

\section{STABILITY ANALYSIS: THE TRANSIENT SOLUTION}

As in previous cases we investigate the stability of the steady state by examining the conditions under which a small perturbation in the temperature field can grow exponentially with time. Such a perturbation will generally only be possible for certain eigenvalues of the exponential growth rate. Stability will be maintained if the growth rates of all such perturbations are non-positive. In cases where complex growth rates are possible, the real part should be non-positive. Otherwise the system will be unstable.

\subsection{Temperature distribution in the rods}

The perturbation in temperature which satisfies both the heat conduction equation and the boundary conditions of constant wall end temperatures is [6]:

where

$$
T_{i}\left(x_{i}\right)=B_{i} \mathrm{e}^{\mu t} \sinh \left(\lambda_{i} x_{i}\right) ; \quad i=1,2,
$$

$$
\lambda_{i}=\sqrt{\frac{a}{k_{i}}}
$$

and $x_{i}(i=1,2)$ is measured in each case from the fixed end.

The perturbation in the temperature difference between the free ends of the rods is then

$$
\Delta T=\mathrm{e}^{a}\left\{B_{1} \sinh \left(y_{1}\right)-B_{2} \sinh \left(y_{2}\right)\right\},
$$

where $y_{i}=i_{i} L_{i}$, and the corresponding perturbation in heat flux is

$$
\Delta Q=-B_{1} K_{1} \lambda_{1} \mathrm{e}^{a t} \cosh \left(y_{1}\right)=B_{2} K_{2} \lambda_{2} \mathrm{e}^{a t} \cosh \left(y_{2}\right)
$$

from considerations of continuity.

The perturbation in the gap, $g$, is then obtained by integrating the free thermal expansion of the rods as

$$
\Delta g=-\frac{B_{1} \alpha_{1}}{i_{1}} \mathrm{e}^{a t}\left\{\cosh \left(y_{1}\right)-1\right\}-\frac{B_{2} \alpha_{2}}{i_{2}} \mathrm{e}^{a t}\left\{\cosh \left(y_{2}\right)-1\right\} .
$$




\subsection{Contact resistance equation}

The relation between the heat flux, $Q$, and the temperature difference, $T$, at the interface can be written

$$
Q=\frac{T f}{S(1-f)}
$$

using equation (4), and hence small linear perturbations about the steady state are related by the equation

i.e.,

$$
\Delta Q=\frac{f \Delta T}{S(1-f)}+\frac{T f^{\prime} \Delta g}{S(1-f)^{2}}
$$

$$
S(1-f) \Delta Q=f \Delta T+\left(T_{1}-T_{2}\right) f^{\prime} \Delta g,
$$

where we have used the fact that, in the steady state, the temperature difference $T=T_{1}^{0}-T_{2}^{0}$ $=(1-f)\left(T_{1}-T_{2}\right)$ from equations (1) and (4).

\subsection{Characteristic equation}

Finally, substituting equations (11)-(13) into (16), we obtain the characteristic equation

$$
\left(T_{1}-T_{2}\right) f^{\prime}\left\{\alpha_{1} L_{1} R_{1} \omega\left(y_{1}\right)-\alpha_{2} L_{2} R_{2} \omega\left(y_{2}\right)\right\}=S(1-f)+f\left\{R_{1} \phi\left(y_{1}\right)+R_{2} \phi\left(y_{2}\right)\right\},
$$

where

$$
\omega(y)=\frac{\cosh (y)-1}{y^{2} \cosh (y)} ; \quad \phi(y)=\frac{\tanh (y)}{y} .
$$

Instability will be predicted whenever equation (17) has one or more zeros corresponding to values of $a$ with positive real part. It can be shown that there are no such zeros when $T_{1}=T_{2}$, i.e. when the temperatures of the two walls are the same. If we start from this condition, in which all the zeros of (17) are therefore in the negative half-plane for $a$, and slowly vary the temperature difference, $\left(T_{1}-T_{2}\right)$, the stability boundary will be defined by the condition when the first zero crosses the imaginary axis, or reaches the positive real axis by passing through the origin.

Unfortunately, the authors have been unable to solve for this condition in closed form in the general case, but some insight into the behaviour of the system can be obtained by examining some special cases.

\subsection{Real zeros}

We first examine the conditions under which equation (17) permits a real positive solution for $a$, in which case $y_{1}, y_{2}$ will be real and positive. There is no loss of generality in choosing the notation in such a way that

$$
r \equiv \frac{y_{1}}{y_{2}}=\frac{L_{1}}{L_{2}} \sqrt{\frac{k_{2}}{k_{1}}}>1 .
$$

The functions $\omega(y), \phi(y)$ take the values $0.5,1$ respectively at $y=0$ and decrease monotonically with $y$, approaching zero as $y \rightarrow \infty$. If $a$ is very small and positive, $y_{1}, y_{2} \ll 1$ and equation (17) can be approximated by the condition

$$
\frac{1}{2} \alpha L\left(T_{1}-T_{2}\right) f^{\prime}=1
$$

Equation (20) defines the limiting value of the temperat ure difference, $\left(T_{1}-T_{2}\right)$, at which a zero passes through the origin onto the positive half-line. It is the first derivative with respect to $g$ of the steady-state equation (4) and, as in previous one-dimensional models, it can be used to conclude that when there are three steady-state solutions, the middle one will be unstable. The reader is referred to reference [6] for more details of this argument.

The derivative $f^{\prime}(g)<0$, in view of the assumed monotonic variation of contact resistance, $R$, with $g$. It therefore follows that instability can only be precipitated by a zero passing into the unstable half-plane through the origin if the direction of heat flow is such that

$$
\alpha L\left(T_{1}-T_{2}\right)<0 \text {. }
$$


This is also the direction required for the existence of multiple solutions [see condition (8)]. However, we shall now demonstrate that zeros can appear on the real axis for the opposite direction of heat flow under certain conditions.

To demonstrate this, we write equation (17) in the form

$$
T_{1}-T_{2}=\frac{S(1-f)+f\left\{R_{1} \phi\left(y_{1}\right)+R_{2} \phi\left(y_{2}\right)\right\}}{f^{\prime}\left\{\alpha_{1} L_{1} R_{1} \omega\left(y_{1}\right)-\alpha_{2} L_{2} R_{2} \omega\left(y_{2}\right)\right\}} .
$$

The denominator of the right hand side of this equation tends to zero as $a \rightarrow \infty$, but it will also be zero at some finite value of $a$ if

$$
\frac{\omega\left(y_{1}\right)}{\omega\left(y_{2}\right)}=\frac{\alpha_{2} L_{2} R_{2}}{\alpha_{1} L_{1} R_{1}}
$$

Now it can be shown from the properties of the function $\omega(y)$ that the ratio $\omega\left(y_{1}\right) / \omega\left(y_{2}\right)$ varies monotonically between 1 and $\left(1 / r^{2}\right)$ as $a$ varies from zero to infinity. Thus, there will always be a single solution of equation (23) if $\left(\alpha_{2} L_{2} R_{2} / \alpha_{1} L_{1} R_{1}\right)$ lies in the range

$$
1>\frac{\alpha_{2} L_{2} R_{2}}{\alpha_{1} L_{1} R_{1}}>\frac{1}{r^{2}}
$$

The conditions (24) are equivalent to the two inequalities

$$
\alpha_{1} L_{2} R_{2}<\alpha_{1} L_{1} R_{1} ; \quad \gamma_{2}>\gamma_{1},
$$

where $\gamma=\alpha / \rho c$ is a material property with dimensions (stress) ${ }^{-1}$. We note that the dimensionless parameter $H=E \gamma /(1-v)$, which arises in the formulation of transient thermoelastic contact problems [14-16], is found to be of the order unity for many engineering materials [14]. Thus, $\gamma$ is generally of the order $E^{-1}$. Values of $\gamma$ and $H$ for some typical engineering materials are given in Table 1.

If the conditions (24) and (25) are satisfied, equation (22) will define a curve of the form shown in Fig. 2, in which $a_{0}$ corresponds to the root of equation (23). In particular, note that there is some critical value, $B$, such that for $\left(T_{1}-T_{2}\right)>B$, there are two positive real roots of the characteristic equation, indicating that the steady-state solution is unstable, despite the fact that it must be unique for this direction of heat flow [see condition (8)].

Furthermore, these zeros of the characteristic equation cannot reach the real axis by passing through the origin, in view of equation (20), and hence must cross the imaginary axis, implying the existence of growing oscillatory solutions in part of the range $0<\left(T_{1}-T_{2}\right)$ $<B$.

Table 1. Properties of Some metals and ceramics

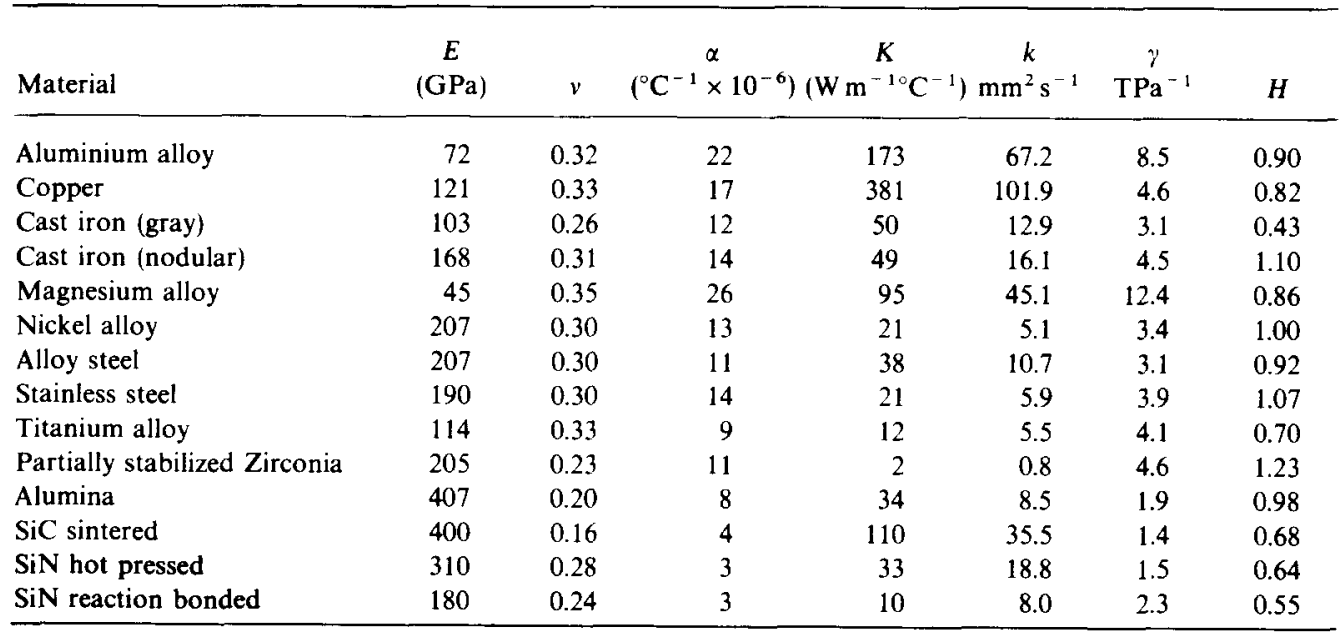




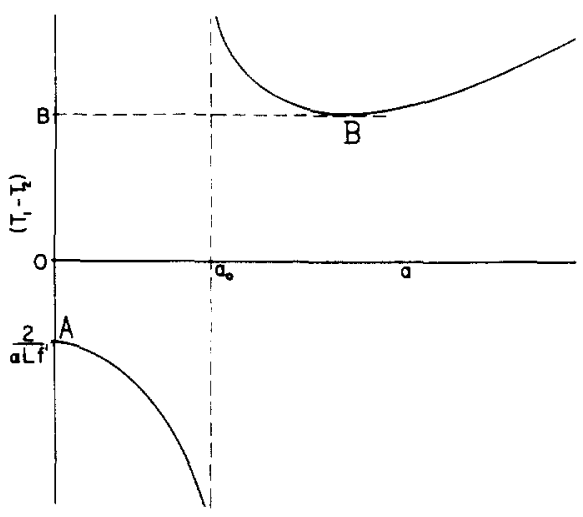

FIG. 2. Form of the r.h.s. of equation (22) as a function of $a$, for the case when equation (23) has a zero at $a=a_{0}$.

Somewhat similar arguments can be applied if the lengths of the rods lie in the range

$$
1<\frac{\alpha_{2} L_{2} R_{2}}{x_{1} L_{1} R_{1}}<r^{2}
$$

In this case, there is no zero in the denominator of the right hand side of equation (22), but it does exhibit a maximum absolute value at some finite $a$, and hence there will be a minimum. $B$, for the expression in the range $0<B<2 / \alpha L f^{\prime}$, where we note that this time $\alpha L<0$ [from equation (26)] and $f^{\prime}<0$.

We conclude that in the range defined by equation (26), there will be zeros on the positive real axis for temperature differences in the range $B<\left(T_{1}-T_{2}\right)<2 / \alpha L f^{\prime}$, i.e. at values lower than that required to produce a zero at the origin. As before, we deduce that these zeros must reach the real axis through unstable complex values, corresponding to unstable oscillatory behaviour, under which conditions the steady state solution is unique but unstable.

\subsection{Complex zeros}

Exponentially growing oscillatory behaviour is obtained if the zeros of equation (17) reach the unstable half-plane by crossing the imaginary axis. We can therefore explore the slability criterion in such cases by postulating the occurrence of a solution $a=\mathrm{i} \xi$, corresponding to $y_{1}=(1+\mathrm{i}) r x, y_{2}=(1+\mathrm{i}) x$, where $r$ is given by (19). The real variable, $x$, can be taken to be positive without loss of generality, since the symmetries of the characteristic equation guarantee that zeros will occur simultaneously at symmetric positions on the four lines $z= \pm(1 \pm \mathrm{i}) x$ in the $y$ plane.

Substituting for $y_{1}, y_{2}$ in equation (17), expanding and equating real and imaginary parts, we obtain the simultaneous equations

where

$$
\begin{aligned}
& \left.f^{\prime}\left(T_{1}-T_{2}\right)\left\{\tilde{F}_{1}^{R}\right\}=S(1-f)+f_{1} \tilde{F}_{2}^{R}\right\} . \\
& f^{\prime}\left(T_{1}-T_{2}\right)\{\not{F}\}=f\{\tilde{F} 2\}
\end{aligned}
$$

$$
\begin{aligned}
& \widetilde{\mathcal{F}}_{1}=\alpha_{1} L_{1} R_{1} \omega(r x+\mathrm{i} r x)-\alpha_{2} L_{2} R_{2}(\omega)(x+\mathrm{i} x) \\
& \widetilde{\mathscr{F}}_{2}=R_{1} \phi(r x+\mathrm{i} r x)+R_{2} \phi(x+\mathrm{i} x)
\end{aligned}
$$

and the superscripts $\mathbf{R}$ and I denote real and imaginary parts, respectively.

Solutions of these equations can be investigated numerically by solving for $f, f^{\prime}$, obtaining

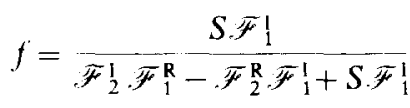

$$
\begin{aligned}
& f^{\prime}\left(T_{1}-T_{2}\right)=f\left(\mathscr{F}_{2} / \mathscr{F}_{1}\right) .
\end{aligned}
$$

We can now evaluate equations (31) and (32) as $x$ traverses the range $0<x<x$. remembering that physically meaningful results require that $0<f<1$. 


\section{SUMMARY OF STABILITY BEHAVIOUR}

An extensive numerical investigation of the conditions for real zeros [equation (22)] and complex zeros [equations (31) and (32)] shows that the system exhibits one of four distinct categories of behaviour, depending upon the value of the ratio

$$
n=\frac{L_{2}}{L_{1}} \sqrt{\frac{k_{1}}{k_{2}}} .
$$

These categories are described qualitatively in the following sections and illustrated graphically for the case of rods of stainless steel $\left(\alpha_{1}=14 \times 10^{-6}{ }^{\circ} \mathrm{C}^{-1}, K_{1}\right.$ $\left.=21 \mathrm{~W} \mathrm{~m}^{-1}{ }^{\circ} \mathrm{C}^{-1}, \quad k_{1}=5.9 \mathrm{~mm}^{2} \mathrm{~s}^{-1}, \quad \gamma_{1}=3.93 \mathrm{TPa}^{-1}\right)$ and aluminium $\left(\alpha_{2}=22\right.$ $\left.\times 10^{-6}{ }^{\circ} \mathrm{C}^{-1}, K_{2}=173 \mathrm{~W} \mathrm{~m}^{-1}{ }^{\circ} \mathrm{C}^{-1}, k_{2}=67 \mathrm{~mm}^{2} \mathrm{~s}^{-1}, \gamma_{2}=8.52 \mathrm{TPa}^{-1}\right)$. When particular materials are under consideration, it is convenient to redefine the notation such that $\gamma_{1}<\gamma_{2}$. This is achieved if rod 1 is of stainless steel and rod 2 is of aluminium, in which case $\gamma_{1} / \gamma_{2}$ $=0.46$.

4.1. $\left(\gamma_{1} / \gamma_{2}\right)^{0.25}<\mathrm{n}<1$

In this case, there is some critical value of $f\left(=f^{*}\right)$, below which the uniqueness and stability criteria exactly coincide-i.e. the steady-state solution is either unique and stable or nonunique and unstable, depending on the value of $f^{\prime}\left(T_{1}-T_{2}\right)$.

If $f>f^{*}$, first complex and then real zeros of the characteristic equation are obtained for temperature differences of the same sign but lower magnitude than that required for nonuniqueness.

The critical value, $f^{*}$, can be found by expanding equation (22) about the point $y=0$ and examining the sign of the second term. If this function is concave towards the axis at small values of $y$, there must be real roots for temperature differences smaller than that given by equation (20). This criterion gives

$$
f^{*}=\frac{5\left(n^{4} \gamma_{2}-\gamma_{1}\right)\left(R_{2}+R_{1}\right)}{4\left(n^{2} \gamma_{2}-\gamma_{1}\right)\left(n^{2} R_{2}+R_{1}\right)}
$$

With rod 1 of stainless steel and rod 2 of aluminium, this behaviour is obtained when 2.78 $<\left(L_{2} / L_{1}\right)<3.37$. Figure 3 shows the case $L_{1}=0.1 \mathrm{~m}, L_{2}=0.29 \mathrm{~m}$, for which $f^{*}=0.417$ and the condition for non-uniqueness is $f^{\prime}\left(T_{1}-T_{2}\right)<-3.20 \times 10^{6}{ }^{\circ} \mathrm{C} \mathrm{m}^{-1}$, bounded by the straight line $\mathrm{U}$ in Fig. 3. The curves $\mathrm{R}$ and $\mathrm{C}$ define the boundaries of real and complex unstable zeros respectively and hence the steady-state solution is unique, but unstable for points between the lines $\mathrm{C}$ and $\mathrm{U}$.

We note from equation (34) that $f^{*} \rightarrow 0$ as $n \rightarrow\left(\gamma_{1} / \gamma_{2}\right)^{0.25}$ and $f^{*} \rightarrow 1.25$ as $n \rightarrow 1$. Thus, there will be a limiting value of $n$, above which $f^{*}>1$ and the uniqueness and stability criteria coincide for all physically acceptable values of $f$. This occurs for stainless steel and aluminium in the range $3.20<\left(L_{2} / L_{1}\right)<3.37$.

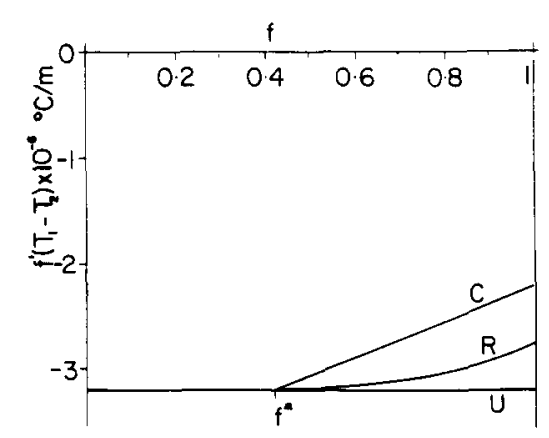

FIG. 3. Stability (C, R) and uniqueness (U) boundaries for $L_{1}=0.1 \mathrm{~m}$ (stainless steel), $L_{2}=0.29 \mathrm{~m}$ (aluminium). 


\section{2. $\left(\gamma_{1} / \gamma_{2}\right)^{0.5}<\mathrm{n}<\left(\gamma_{1} / \gamma_{2}\right)^{0.25}$}

This range corresponds to $2.23<\left(L_{2} / L_{1}\right)<2.78$. Figure 4 shows the corresponding bounding curves for the case $L_{1}=0.1 \mathrm{~m}, L_{2}=0.25 \mathrm{~m}$, for which non-uniqueness occurs when $f^{\prime}\left(T_{1}-T_{2}\right)<-9.69 \times 10^{6}{ }^{\circ} \mathrm{C} \mathrm{m}^{-1}$. Curves $\mathrm{C}$ and $\mathrm{R}$ now fall above $\mathrm{U}$ for all values of $f$, thus defining a domain of unique unstable steady-state solutions.

As $n \rightarrow\left(\gamma_{1} / \gamma_{2}\right)^{0.5}$, the line $\mathrm{U}$ tends to $-\infty$, but the curves $\mathrm{C}, \mathrm{R}$ remain in the finite domain.

\section{3. $0<\mathrm{n}<\left(\gamma_{1} / \gamma_{2}\right)^{0.5}$}

In this range, $0<\left(L_{2} / L_{1}\right)<2.23$ and multiple solutions require the opposite direction of heat flow $\left(T_{2}>T_{1}\right)$ as illustrated in Fig. 5 for the case $L_{1}=0.1 \mathrm{~m}, L_{2}=0.2 \mathrm{~m}$. Multiple solutions occur for $f^{\prime}\left(T_{1}-T_{2}\right)>7.49 \times 10^{6}{ }^{\circ} \mathrm{Cm}^{-1}$.

The criterion for real roots defines two boundary lines, denoted by $R_{A}$ and $R_{B}$ in Fig. 5 and corresponding to the points $\mathrm{A}$ and $\mathrm{B}$ respectively in Fig. 2. The uniqueness boundary, $\mathrm{U}$, coincides with $R_{A}$. The solution is unique and stable in the region between the curves $C$ and $\mathrm{U}$. Beyond $\mathrm{C}$ it is unstable and unique, whereas beyond $\mathrm{U}$ it is unstable and non-unique.

\section{4. $n>1$}

For rods of stainless steel and aluminium, this condition is satisfied if $\left(L_{2} / L_{1}\right)>3.37$. No complex roots are obtained and the lowest real root coincides with the criterion for nonuniqueness. Thus the steady-state solution is either unique and stable or non-unique and unstable, as in the more elementary one rod models [6-8].

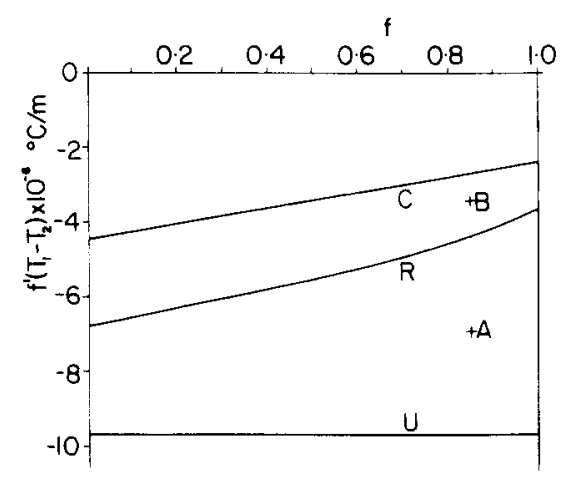

F1G. 4. Stability and uniqueness boundaries for $L_{1}=0.1 \mathrm{~m}$ (stainless steel). $L_{2}=0.25 \mathrm{~m}$ (aluminium).

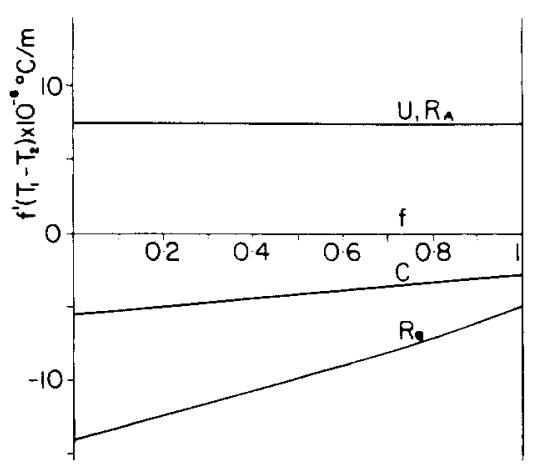

FIG. 5. Stability and uniqueness boundaries for $L_{1}=0.1 \mathrm{~m}$ (stainless steel), $L_{2}=0.20 \mathrm{~m}$ (aluminium). 


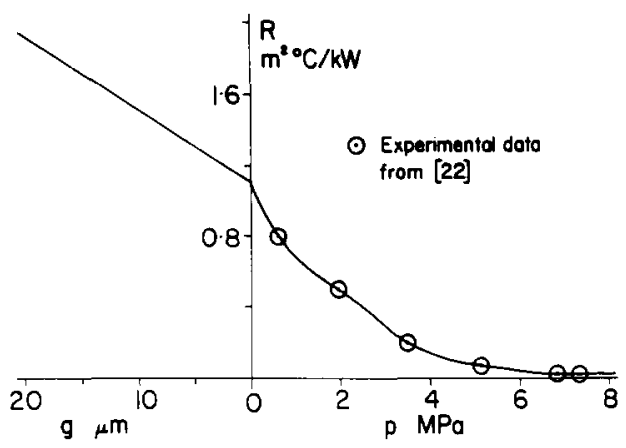

FiG. 6. Relation between thermal resistance, $R$, and contact pressure, $p$, or gap, $g$.

\section{NUMERICAL INVESTIGATION OF TRANSIENT BEHAVIOUR}

The foregoing analysis is based on a linear perturbation of the system and is valid only in the immediate vicinity of the steady state. It therefore enables us to determine the stability of the various steady-state solutions but not to predict the subsequent transient behaviour in cases of instability. This is a question of particular interest when the system has a unique steady-state solution which is unstable, for which the long term behaviour must presumably be either periodic and regular or quasi-chaotic.

\subsection{The finite difference algorithm}

The full transient problem is not analytically tractable, but, being one-dimensional, it is a fairly straightforward matter to model its behaviour numerically using the finite difference method.

The instantaneous temperature field in each rod is represented by the temperatures at $N$ equally spaced points, including the end points. The instantaneous values of these temperatures can then be used to calculate the free thermal expansion of the rods and hence the gap or contact pressure between the free ends.

The heat flux, $Q$, across the interface is then calculated on the assumption that the interface thermal resistance, $R$, is a given function of contact pressure or gap (see section 5.2 below). This heat flux is taken to be constant during the subsequent small time increment, thus permitting an explicit algorithm to be used for updating the temperature values.

The temperatures at interior points are updated using equations (4) and (5) of Ref. [17], $\$ 18.3$, and the temperatures at the fixed ends of the rods are the prescribed wall temperatures $T_{1}$ and $T_{2}$. The flux boundary condition at the interface ends of the rods is satisfied using Schmidt's method [see [17], $\$ 18.4$, equation (4)], in which a fictitious point is defined beyond the end of each rod.

\subsection{The contact resistance function}

The interface resistance for two bodies in contact has been the subject of extensive experimental and theoretical study [18-23]. It is known to be very sensitive to contact pressure, at least when the nominal contact pressure is small in comparison with the material hardness, so that the ratio between actual and nominal contact area is small. It is also affected by the roughness of the contacting surfaces and the thermal properties of the contacting bodies and the intervening gas.

For the present study, we investigate the contact of rods of aluminium and stainless steel, for which appropriate experimental data are given by Thomas and Probert [22]. The corresponding surface condition for these data is given in Table 1 of Ref. [22] (specimens AL3 and EM1').

Experimental data are not available for the case where a gap exists between the surfaces, but a reasonable estimate can be obtained in the form

$$
R(g)=R(0)+g / K_{\text {air }},
$$


where $R(0)$ is the thermal resistance corresponding to contact at zero pressure and $K_{\text {air }}$ $\left(=2.43 \times 10^{-2} \mathrm{~W} \mathrm{~m}^{-1}{ }^{\circ} \mathrm{C}^{-1}\right)$ is the conductivity of air, which is taken to occupy the space between the surfaces. The value $R(0)$ is obtained by extrapolation from the data at finite pressure.

Figure 6 shows the resulting relation between resistance and contact pressure or gap. including the experimental data points from [22]. We note in particular the sensitivity of resistance to pressure in lightly loaded contacts (e.g. $0<p<4 \mathrm{MPa}$ ).

\subsection{Choice of time step}

The accuracy and numerical stability of the algorithm depends upon the use of a sufficiently small time step. However, the usual criterion for numerical stability in the finite difference method [condition (7), [17], $\$ 18.3$ ] proved to be inadequate here because of the extreme sensitivity of the interface resistance to small changes in pressure and hence temperature. The resulting numerical instabilities are characterized by divergent oscillations in time on the scale of the discretization. In other words, the temperature at one or more points near the interface alternately increases and decreases in successive time increments with increasing amplitude.

This difficulty can be overcome by using an extremely small time step, but the resulting volume of computations becomes prohibitive in many cases. Fortunately, numerical instabilities associated with variations in contact resistance are only generated at certain stages during the evolution of the system. It was therefore possible to develop a more efficient algorithm in which the results are monitored for the onset of numerical instability and adaptive changes in the time step are made as required.

The three previous values of temperature at each point are stored, and if two consecutive reversals of temperature gradient are detected at any point, the system is set back to its state before the instability began and the time step is reduced. A reduction in time step is also triggered if the temperature at any point changes by more than $1 \%$ in any time increment. A related mechanism permits the time step to increase gradually during periods of stable computation.

The threshold values for this adaptive time step variation were chosen so as to ensure convergence of the results on a unique system history within $0.1 \%$. The satisfaction of this criterion was demonstrated for an extensive range of system parameters -indeed, in most circumstances, convergence accuracy was better than $0.01 \%$ at all temperatures.

\section{NUMERICAL RESULTS}

Initially the system was set at a steady state, for which the temperature variation along the rods is linear and can be calculated from the equations given in section 2. A small temperature perturbation was then applied to induce a transient response. Typically the temperature at one interior node near the interface in each rod was changed from the steady-state value by a small quantity of the order $\left(\left|T_{2}-T_{1}\right| / 1000\right)$.

In the initial evolution of the system it is far from obvious whether the behaviour is stable or unstable. It is therefore advantageous to choose an initial perturbation which minimizes the time taken to return to the steady state in cases of stability. This can be done by ensuring that the increase in the total heat stored in one rod is balanced by a corresponding decrease in heat stored in the other. In other words, the total heat stored in the two rods after the perturbation is the same as in the steady state. If a net change in total heat stored were introduced, the system could only return to the steady state when the excess heat had had time to flow through the rods to one or other of the walls.

The analysis of stability summarized in section 4 above shows that instability is more likely to occur when $f^{\prime}$ is large--i.e. when the initial gap, $g_{0}$, is chosen so as to give a steady state in the range where the interface resistance is very sensitive to small changes in pressure or gap. This is typically the case when the steady state involves contact at low pressure (see Fig. 6). 


\subsection{Convergence on stable solutions}

Several tests of the program were made with parameter values for which the steady-state solution was predicted to be stable. In all cases, the temperatures returned monotonically to the steady state after the initial perturbation as anticipated.

A more interesting case arises when the steady-state solution is non-unique. Figure 7 shows the development of the temperature of the free ends of the rods for the case $L_{1}=0.1 \mathrm{~m}$, $L_{2}=0.29 \mathrm{~m}, T_{1}=0^{\circ} \mathrm{C}, T_{2}=350^{\circ} \mathrm{C}$. With a suitable choice of $g_{0}$, these values correspond to a point below the line $\mathrm{U}$ in Fig. 3 and hence to an unstable but non-unique initial condition. The system diverges initially, passes through one cycle of oscillation, but eventually converges on one of the other two steady states, both of which can be shown to be stable by the criteria of section 4 , since they correspond to conditions in which the contact resistance is less sensitive to variations in pressure and gap respectively and hence to lower values of $\left(-f^{\prime}\right)$. The initial perturbation used in this case $\left(5 \times 10^{-5}{ }^{\circ} \mathrm{C}\right)$ is too small to make a measurable difference at $t=0$ in Fig. 7.

\subsection{Oscillatory behaviour}

When the system parameters correspond to a condition in which the steady-state solution is unstable but non-unique, the system always exhibits a non-linear oscillatory behaviour. Typical results are shown in Fig. 8 for the case $L_{1}=0.1 \mathrm{~m}, L_{2}=0.25 \mathrm{~m}, T_{1}=0^{\circ} \mathrm{C}, T_{2}$ $=400^{\circ} \mathrm{C}$, corresponding to the point $\mathrm{A}$ in Fig. 4 . With a small initial perturbation, the system takes about 10 minutes to diverge sensibly from the initial steady state, but after one or two cycles it settles into a very reproducible oscillatory state with a period of 35 minutes. This period is of the same order as that taken for a thermal disturbance to propagate to the walls from the interface. Thus, defining a Fourier number,

$$
F_{0}=k t / L^{2},
$$

we find that $F_{0}=1$ would correspond to times of 28 seconds and 15 seconds for the steel and aluminium rods respectively.

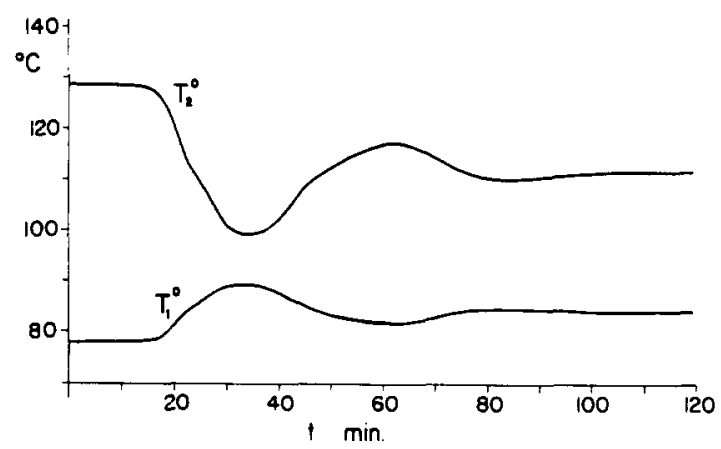

FIG. 7. Temperatures at the free ends of the rods for $L_{1}=0.1 \mathrm{~m}, L_{2}=0.29 \mathrm{~m}, T_{1}=0^{\circ} \mathrm{C}, T_{2}=350^{\circ} \mathrm{C}$.

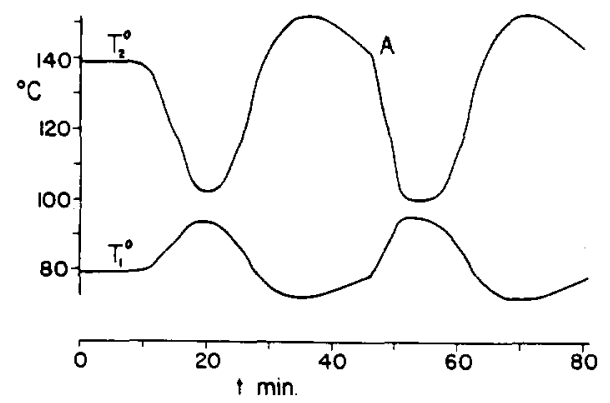

FIG. 8. Temperatures at the free ends of the rods for $L_{1}=0.1 \mathrm{~m}, L_{2}=0.29 \mathrm{~m}, T_{1}=0^{\circ} \mathrm{C}, T_{2}=400^{\circ} \mathrm{C}$ (point A of Fig. 4). 
The corresponding variation in contact pressure and gap at the interface is shown in Fig. 9 and we note that the discontinuity in slope in Fig. 8 (point $\mathrm{A}$ ) is associated with the transition from separation to contact.

If the temperature $T_{2}$ is reduced, the corresponding point in Fig. 4 moves towards the stability boundary and the amplitude of the temperature oscillation is reduced. Figures 10 and 11 show respectively the free end temperatures and the contact pressure/gap for $T_{2}$ $=200^{\circ} \mathrm{C}$, corresponding to the point B in Fig. 4. The period of the oscillations is increased slightly to 44 minutes, but there is a more substantial increase in the time taken to establish the steady oscillatory behaviour. This is to be anticipated, since the closer we approach the stability boundary, the slower will be the exponential growth rate of the corresponding perturbation.

This causes numerical difficulties when conditions are chosen to be very close to the stability boundary, since the changes in temperature in one time step at the beginning of the process may be less than the rounding error, thus causing the system to lock into a spurious numerically-generated steady state. This difficulty can be alleviated by using a larger initial perturbation or by starting the system in a non-steady state, but in general the numerical algorithm is not well suited to conditions in which the changes in temperature are very gradual.

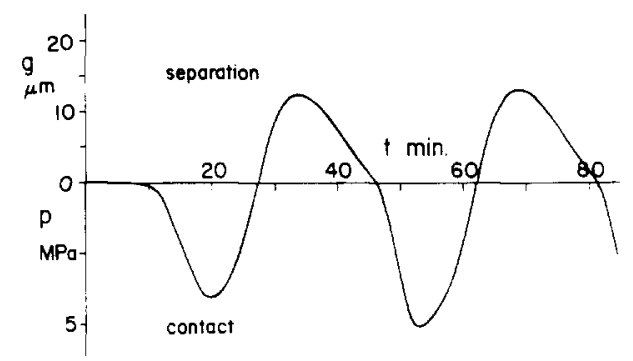

FiG. 9. Variation of contact pressure, $p$, and gap, $y$, for the conditions of Fig. 8

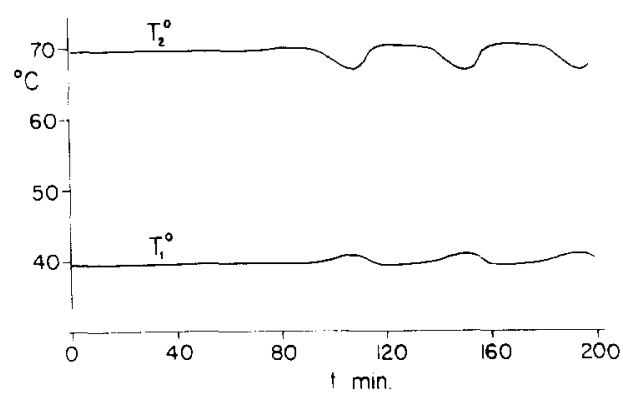

Fis. 10. Temperatures at the free ends of the rods for $L_{1}=0.1 \mathrm{~m}, L_{2}=0.29 \mathrm{~m}, T_{1}=0 \mathrm{C}$ $T_{2}=200^{\circ} \mathrm{C}$ (point B of Fig. 4).

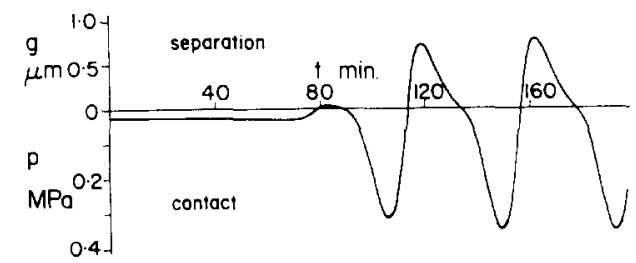

FIG. 11. Variation of contact pressure, $p$, and gap, $g$, for the conditions of Fig. 10 


\section{CONCLUSIONS}

It is clear from the present paper that the stability behaviour of systems involving the contact of two thermoelastic bodies is considerably more complex than had been suspected from earlier analyses involving a single body and a rigid wall. In particular, it is not possible to define the stability criterion in terms of the minimization of an energy function, since stability now depends upon the thermal diffusivities of the materials as well as those properties like thermal conductivity, which govern the steady-state solutions.

A substantial range of conditions is found in which the system has a unique steady-state solution which is unstable. A numerical analysis of the system demonstrates that in such conditions it always tends to a steady oscillatory state in which the contact pressure varies periodically with time, possibly with periods of separation.

These conclusions have important implications for the classical steady-state solutions to two- and three-dimensional thermoelastic contact problems such as the Hertzian contact of spheres at different temperatures [12]. Since the uniqueness of such solutions cannot now be regarded as a guarantee of stability, their stability needs to be re-examined. In particular, we must accept the possibility that a steady oscillatory state exists. The possibility of oscillatory behaviour in thermoelastic contact has been discussed ever since difficulties with existence were first discovered with the classical boundary conditions [1]. Indeed, Clausing [24] reported slow periodic variations in experimental measurements of thermal contact resistance which may be attributable to this mechanism. Thus, this paper opens up an extensive field for further investigation of the phenomenon of thermoelastic contact.

Acknowledgements-The authors are pleased to acknowledge support from the National Science Foundation under Contract number MSM-8419324 and from the Aluminium Company of America.

\section{REFERENCES}

1. J. R. BARBER, The effect of thermal distortion on construction resistance. Int. J. Heat Mass Transfer 14, 751-766 (1971).

2. M. Comninou and J. Dundurs, On the Barber boundary conditions for thermoelastic contact. J. appl. Mech. 46, 849-853 (1979).

3. J. Dundurs and M. Comninou, On the boundary conditions in contact problems with heat conduction. In Developments in Theoretical and Applied Mechanics, 8 (Edited by R. P. MCNITT), pp. 3-11. Virginia Polytechnic Institute and State University (1976).

4. J. R. BARBER, Contact problems involving a cooled punch. J. Elasticity 8, 409-423 (1978).

5. G. Duvaut, Free boundary problem connected with thermoelasticity and unilateral contact. In Free Boundary Problems, Vol. II. Pavia (1979)

6. J. R. BARBER, J. Dundurs and M. Comninou, Stability considerations in thermoelastic contact. J. appl. Mech. 47, $871-874$ (1980).

7. J. R. BARBER, Stability of thermoelastic contact for the Aldo model. J. appl. Mech. 48, 555-558 (1981)

8. J. B. Barber, Non-uniqueness and stability for heat conduction through a duplex heat exchanger tube. $J$. Thermal Stresses 9, 69-78 (1986).

9. J. R. BARBER, Stability of thermoelastic contact. Institution of Mechanical Engineers, International Conference on Tribology, pp. 981-986. London (1987).

10. T. A. Dow and R. A. BURTON, Thermoelastic instability of sliding contact in the absence of wear. Wear 19 315-328 (1972).

11. O. Richmond and N. C. HuANG, Interface stability during unidirectional solidification of a pure metal. Proc. 6th Canadian Congr. appl. Mech., pp. 453-454. Vancouver (1977).

12. J. R. BARBer, Indentation of the semi-infinite elastic solid by a hot sphere. Int. J. Mech. Sci. 15, 813-819 (1973).

13. M. Comninou and J. Dundurs, On the possibility of history dependence and instabilities in thermoelastic contact. J. Thermal Stresses 3, 427-433 (1980).

14. D. A. HILLS and J. R. BARBER, Steady motion of an insulating rigid flat-ended punch over a thermally conducting half-plane. Wear 102, 15-22 (1985).

15. D. A. HILLS and J. R. BARBER, Steady sliding of a circular cylinder over a dissimilar thermally conducting halfplane. Int. J. Mech. Sci. 28, 613-622 (1986).

16. H. BLOK, Thermo-tribology-fifty years on. Institution of Mechanical Engineers, International Conference on Tribology, pp. 1-8. London (1987).

17. H. Carslaw and J. C. Jaeger, The Conduction of Heat in Solids, 2nd edn. Clarendon Press, Oxford (1959).

18. M. E. BARZELAY, K. E. TONG and G. F. HollowaY, Effect of pressure on thermal conductance of contact joints. NACA TN 3295 (1955). 
19. YU. P. Shlykov and YE. A. Ganin, Thermal resistance of metallic contacts. Int. J. Heat Mass Transfer 7. 921-929 (1964).

20. A. M. CLausing and B. T. ChaO, Thermal contact resistance in a vacuum environment. ASME J. Heat Transfer 87, 243-251 (1965).

21. M. G. CoOper, B. B. Mikic and M. M. Yovanovich, Thermal contact conductance. Int. J. Heat Mass Transfer 12, 279 (1969).

22. T. R. Thomas and S. D. Prober T, Thermal contact resistance: The directional effect and other problems. Int. $J$. Heat Mass Transfer 13, 789-807 (1970).

23. B. B. MikiC, Thermal contact conductance: Theoretical considerations. Int. J. Heat Mass Transfer 17, 205 (1974). 